\title{
An analogue of the Riesz theorem in Hardy-Morrey classes
}

\author{
Miran I. Aleskerov *
}

Received: 18.07.2019 / Revised: 19.01.2020 / Accepted: 06.02.2020

\begin{abstract}
In this paper study the Hardy-Morrey classes of analytic functions inside and outside the unit disk generated by the norm of the Morrey space on the unit circle. Since the Morrey space is not separable, therefore, the analogue of the Riesz theorem in these classes differs from the classical version.
\end{abstract}

Keywords. Hardy-Morrey classes, Morrey space, Riesz theorem.

Mathematics Subject Classification (2010): 33B10, 46E30, 54D70

\section{Introduction}

Morrey space was introduced in connection with the study of the smoothness properties of the solution of an elliptic equation with coefficients from the BMO (boundary mean oscillation) space in 1938 by S. Morrey. Recently, interest in the study of various problems of mathematics in these spaces has greatly increased. Many problems of harmonic analysis are studied in Morrey spaces in the works of various mathematicians (see e.g. [2-5, 7-14, $17,19])$. More detailed about related issues can be found in monographs $[1,15,16]$. Morrey spaces are not separable. Therefore, approximation problems in these spaces have their own characteristics. In [2-5], subspaces of Morrey spaces generated by a shift operator in which continuous functions are dense are distinguished. Applying the method of Riemann boundary value problems in these works, the basic properties of some perturbed trigonometric systems in these subspaces are studied. In this case, the solvability of the Riemann boundary value problem in Hardy-Morrey classes is essentially used. It should be noted that the Riesz theorem plays a key role in theory of Hardy classes.

In this paper Hardy-Morrey classes of analytic functions inside and outside the unit disk generated by the norm of the Morrey space on the unit circle. Since the Morrey space is not separable, therefore, the analogue of the Riesz theorem in these classes differs from the classical version.

\section{Needful information}

In this section we state some notations and facts which will be used to obtain our main results. Let's first define the Morrey space on the unit circle $\gamma=\{z \in C:|z|=1\}$ on the

\footnotetext{
* Corresponding author
}

M.I. Aleskerov

Ganja State University, Ganja, Azerbaijan

E-mail: miran.alesgerov@mail.ru 
complex plane $C$. Next, $\omega=$ int $\gamma$ will denote the unit ball in $C$. By $L_{0}(-\pi, \pi)$ we denote the linear space of all (Lebesgue-) measurable functions on $(-\pi, \pi)$.

$L^{p, \alpha}(\gamma), 1 \leq p<+\infty, 0 \leq \alpha \leq 1$, will denote the normed space of all measurable functions $f(\cdot)$ on $\gamma$ with the finite norm

$$
\|f\|_{L^{p, \alpha}(\gamma)}=\sup _{B}\left(|B \bigcap \gamma|_{\gamma}^{\alpha-1} \int_{B \bigcap \gamma}|f(\xi)|^{p}|d \xi|\right)^{1 / p}<+\infty
$$

$\left(|B \bigcap \gamma|_{\gamma}\right.$-is the linear measure of intersection $\left.B \bigcap \gamma\right)$, where sup is taken over all balls centered at $\gamma$ with an arbitrary positive radius. $L^{p, \alpha}(\gamma)$ is a Banach space with respect to this norm. We also define the space $L^{p, \alpha}(-\pi, \pi), 1 \leq p<+\infty, 0 \leq \alpha \leq 1$, which consists of measurable functions $f(\cdot)$ on $(-\pi, \pi)$ with the finite norm

$$
\|f\|_{L^{p, \alpha}(-\pi, \pi)}=\sup _{I \subset[-\pi, \pi]}\left(|I|^{\alpha-1} \int_{I}|f(t)|^{p}|d t|\right)^{1 / p}<+\infty
$$

where sup is taken over all intervals $I \subset[-\pi, \pi]$. It is not difficult to see that the correspondence $f(t)=: F\left(e^{i t}\right), t \in(-\pi, \pi), F(\cdot) \in L^{p, \alpha}(\gamma)$ establishes an isometric isomorphism between the spaces $L^{p, \alpha}(\gamma)$ and $L^{p, \alpha}(-\pi, \pi)$. Therefore, in what follows we will equate these spaces and denote $L^{p, \alpha}$ with the norm $\|\cdot\|_{p, \alpha}$.

It is not difficult to see that for $0 \leq \alpha_{1} \leq \alpha_{2} \leq 1$ the following continuous embedding holds: $L^{p, \alpha_{1}} \subset L^{p, \alpha_{2}}$. Moreover, it is clear that $L^{p, 1}=L_{1}(-\pi, \pi)$ and $L^{p, 0}=L_{\infty}(-\pi, \pi)$. We also have $L^{p, \alpha} \subset L_{1}(-\pi, \pi), \quad \forall \alpha \in[0,1], \forall p \geq 1$.

It is valid the following

Lemma 2.1 [2] The space $L_{\infty}$ (and so $C[-\pi, \pi]$ too) is not dense in $L^{p, \alpha}$ for $1 \leq p<$ $+\infty$ and $\forall \alpha \in(0,1)$.

It follows that the sequence of bounded functions cannot be complete in $L^{p, \alpha}$. In what follows, we will assume, if needed, that the function $f \in L^{p, \alpha}$ is periodically (with period $2 \pi$ ) extended to the whole real axis $R$. Following Lemma 2.1, we will consider the subspace $M^{p, \alpha}$ of functions $f(\cdot)$ the shifts of which are continuous in $L^{p, \alpha}$, i.e. $\|f(\cdot+\delta)-f(\cdot)\|_{p, \alpha} \rightarrow$ $0, \quad \delta \rightarrow 0$.

Lemma 2.2 [2] The space $M^{p, \alpha}, 1 \leq p<+\infty, 0<\alpha \leq 1$, is Banach and $C_{0}^{\infty}[-\pi, \pi]$ is dense in it.

Define the Morrey-Hardy class $H_{+}^{p, \alpha}, 1 \leq p<+\infty, 0 \leq \alpha \leq 1$, of functions $f(\cdot)$ analytic inside $\omega$ endowed with the norm

$$
\|f\|_{H_{+}^{p, \alpha}}=\sup _{0<r<1}\left\|f_{r}(\cdot)\right\|_{p, \alpha},
$$

where $f_{r}(t)=f\left(r e^{i t}\right)$. It is not difficult to see that the inclusion $H_{+}^{p, \alpha} \subset H_{1}^{+}, \quad 1 \leq p<$ $+\infty$, holds, where $H_{1}^{+}-$is a usual Hardy class. Therefore, every function $f(\cdot) \in H_{+}^{p, \alpha}$ has non-tangential boundary values $f^{+}(\cdot)$ on $\gamma$.

Also let

$$
M H_{+}^{p, \alpha}=\left\{F \in H_{+}^{p, \alpha}: F^{+} \in M^{p, \alpha}\right\} .
$$

Absolutely similar to the classical case, we define the Morrey-Hardy class outside the unit circle $\omega$. Let $\omega^{-}=C \backslash \bar{\omega}(\bar{\omega}=\omega \bigcup \gamma)$. We will say that the function $f$ analytic in $\omega^{-}$has 
a finite order $m$ at infinity, if its Laurent decomposition at infinitely remote point has the following form

$$
f(z)=\sum_{k=-\infty}^{m} a_{k} z^{k}, \quad a_{m} \neq 0 .
$$

Thus, for $m>0$ function $f$ has a pole of order $m$ at $z=\infty$; for $m=0$ it is bounded in the vicinity of $z=\infty$; and in case $m<0$ it has a zero of order $(-m)$ at $z=\infty$. Let $f(z)=f_{0}(z)+f_{1}(z)$, where $f_{0}(z)$ is a principal part (i.e. $f_{0}(z)=\sum_{k=0}^{m} a_{k} z^{k}$ ), and $f_{1}(z)$ regular part of decomposition (2.1). Consequently, $f_{0}(z) \equiv 0$ for $m<0$ and $f_{0}$ is a polynomial of order $m$, i.e. $\operatorname{deg} f_{0}=m$, if $m \geq 0$. We say that the function $f$ belongs to the class ${ }_{m} H_{-}^{p, \alpha}$, if $\operatorname{deg} f_{0} \leq m$ and $F \in H_{+}^{p, \alpha}$, where $F(z)=\overline{f_{1}\left(\frac{1}{\bar{z}}\right)}, \quad z \in \omega$.

Similarly we define

$$
{ }_{m} M H_{-}^{p, \alpha}=\left\{F \in{ }_{m} H_{-}^{p, \alpha}: F^{-} \in M^{p, \alpha}\right\} .
$$

We also need the following Riesz-Fichtenholz theorem.

Theorem R-F. In order to the function $\Phi(\cdot)$ belong to the class $H_{1}$, it is necessary and sufficient that it can be represented as the Poisson-Stieltjes integral

$$
\begin{gathered}
\left(z=\rho e^{i \sigma}\right) \\
\Phi(z)=\frac{1}{2 \pi} \int_{-\pi}^{\pi} \mathcal{P}(\rho, \sigma-s) d \mu(s),
\end{gathered}
$$

or in the form of a Poisson-Lebesgue integral

$$
\Phi(z)=\frac{1}{2 \pi} \int_{-\pi}^{\pi} \Phi^{+}\left(e^{i s}\right) \mathcal{P}(\rho, \sigma-s) d s,
$$

or in the form of a Cauchy-Lebesgue integral

$$
\Phi(z)=\frac{1}{2 \pi} \int_{-\pi}^{\pi} \frac{\Phi^{+}\left(e^{i s}\right) e^{i s} d s}{e^{i s}-z},
$$

or in the form of a Cauchy-Stieltjes integral

$$
\Phi(z)=\frac{1}{2 \pi} \int_{-\pi}^{\pi} \frac{e^{i s} d \mu(s)}{e^{i s}-z},
$$

where $\mathcal{P}(\cdot, \cdot)$ is the Poisson kernel

$$
\mathcal{P}(\rho, s-\varphi)=\frac{1-\rho^{2}}{1-2 \rho \cos (s-\varphi)+\rho^{2}}=R e \frac{e^{i s}+\rho e^{i \varphi}}{e^{i s}-\rho e^{i \varphi}},
$$

$\mu(\cdot)$ is a function of bounded variation on $[-\pi, \pi]$.

Regarding this theorem, one can see, e.g., monographs $[6,18]$.

In obtaining the main results, we will also use the following properties of the Poisson kernel.

Theorem 2.1 The Poisson kernel $\mathcal{P}(\cdot, \cdot)$ has the following properties:

i) $\frac{1}{2 \pi} \int_{-\pi}^{\pi} \mathcal{P}(r, s) d s=1, \forall r \in[0,1)$;

ii) $\sup |\mathcal{P}(r, s)| \rightarrow 0, r \rightarrow 1-0, \forall \delta>0$;

$|s| \geq \sigma$

iii) $\int_{|s| \geq \delta} \mathcal{P}(r, s) d s \rightarrow 0, r \rightarrow 1-0, \forall \delta>0$. 
An the end of this section an analogue of Minkowski inequality in Morrey space will be given.

Proposition 2.1 Let $F:[-\pi, \pi] \times[-\pi, \pi] \rightarrow C$ be a measurable function and $F(\cdot ; s) \in$ $L^{p, \alpha}, 1 \leq p<+\infty, 0<\alpha<1$, for $\forall s \in[-\pi, \pi]$. Then it is valid

$$
\left\|\int_{-\pi}^{\pi} F(\cdot ; s) d s\right\|_{p, \alpha} \leq \int_{-\pi}^{\pi}\|F(\cdot ; s) d s\|_{p, \alpha} d s .
$$

\section{Main results}

The classical Riesz theorem has the following analogue with respect to the Hardy-Morrey classes.

Theorem 3.1 Let $1 \leq p<+\infty, 0<\alpha<1$.

Then:

$\alpha)\|F(\cdot)\|_{H_{+}^{p, \alpha}}=\left\|F^{+}(\cdot)\right\|_{p, \alpha}, \forall F \in H_{+}^{p, \alpha}\left(\|F(\cdot)\|_{m} H_{-}^{p, \alpha}=\left\|F^{-}(\cdot)\right\|_{p, \alpha}, \forall F \in{ }_{m} H_{-}^{p, \alpha}\right)$;

$\beta)$ For $F \in H_{+}^{p, \alpha}\left(F \in{ }_{m} H_{-}^{p, \alpha}\right)$ the relation $\lim _{r \rightarrow 1-0}\left\|F_{r}(\cdot)-F^{+}(\cdot)\right\|_{p, \alpha}=0$

$\left(\lim _{r \rightarrow 1+0}\left\|F_{r}(\cdot)-F^{-}(\cdot)\right\|_{p, \alpha}=0\right)$ holds if and only if $F(\cdot) \in M H_{+}^{p, \alpha}\left(F(\cdot) \in{ }_{m} M H_{-}^{p, \alpha}\right)$, where $F_{r}(t)=F\left(r e^{i t}\right)$.

Proof. Let us show the validity of $\alpha$ ) with respect to $F \in H_{+}^{p, \alpha}$. The case $F \in{ }_{m} H_{-}^{p, \alpha}$ is proved similarly. So, let $F \in H_{+}^{p, \alpha}$. Then it is clear that $F(\cdot) \in H_{p}^{+}$, where $H_{p}^{+}$is a usual Hardy class. By the classical Riesz theorem, we have

$$
\int_{-\pi}^{\pi}\left|F\left(r e^{i t}\right)-F^{+}\left(e^{i t}\right)\right|^{p} d t \rightarrow 0, r \rightarrow 1-0 .
$$

Hence we obtain that $\exists\left\{r_{n}\right\}_{n \in N} \subset(0,1): r_{n} \rightarrow 1-0, n \rightarrow \infty$, such that the sequence $\left\{F\left(r_{n} e^{i t}\right)\right\}_{n \in N}$ converges to $F^{+}\left(e^{i t}\right)$ a.e. $t \in[-\pi, \pi]$. Let $E \subset[-\pi, \pi]-$ be an arbitrary interval. It is clear that $\left|F\left(r_{n} e^{i t}\right)\right|^{p} \rightarrow\left|F^{+}\left(e^{i t}\right)\right|^{p}$, a.e. $t \in E$. We have

$$
\frac{1}{|E|^{1-\alpha}} \int_{E}\left|F\left(r_{n} e^{i t}\right)\right|^{p} d t \leq \sup _{\mathrm{I} \subset[-\pi, \pi]}\left(\frac{1}{|\mathrm{I}|^{1-\alpha}} \int_{\mathrm{I}}\left|F\left(r_{n} e^{i t}\right)\right|^{p} d t\right) \leq\|F\|_{H_{+}^{p, \alpha}}^{p} .
$$

Considering this relation from Fatou's theorem we get

$$
\int_{E}\left|F^{+}\left(e^{i t}\right)\right|^{p} d t \leq \sup _{n} \int_{E}\left|F\left(r_{n} e^{i t}\right)\right|^{p} d t \leq|E|^{1-\alpha}\|F\|_{H_{+}^{p, \alpha}}^{p}, \forall E \subset[-\pi, \pi] .
$$

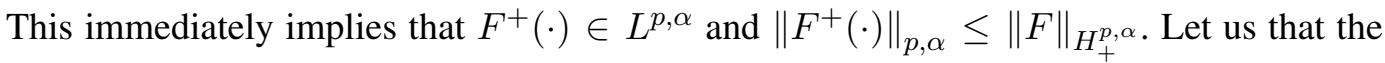
converse inequality also holds. By R-F Theorem, the function $F(\cdot)$ has a representation in the form of a Lebesgue-Poisson integral

$$
F\left(\rho e^{i \sigma}\right)=\frac{1}{2 \pi} \int_{-\pi}^{\pi} F^{+}\left(e^{i s}\right) \mathcal{P}(\rho, \sigma-s) d s=\frac{1}{2 \pi} \int_{-\pi}^{\pi} F^{+}\left(e^{i(\sigma-s)}\right) \mathcal{P}(\rho, s) d s .
$$

We have $\left(F_{r}(t)=F\left(r e^{i t}\right)\right)$

$$
\left\|F_{r}(\cdot)\right\|_{p, \alpha}=\left\|\frac{1}{2 \pi} \int_{-\pi}^{\pi} F^{+}\left(e^{i(t-s)}\right) \mathcal{P}(r, s) d s\right\|_{p, \alpha} \leq
$$




$$
\frac{1}{2 \pi} \int_{-\pi}^{\pi}\left\|F^{+}\left(e^{i(\cdot-s)}\right)\right\|_{p, \alpha} \mathcal{P}(r, s) d s .
$$

It is easy to see that the norm $\|\cdot\|_{p, \alpha}$ is invariant with respect to the shift, i.e.

$$
\left\|F^{+}\left(e^{i(\cdot-s)}\right)\right\|_{p, \alpha}=\left\|F^{+}(\cdot)\right\|_{p, \alpha} .
$$

Then taking into account

$$
\frac{1}{2 \pi} \int_{-\pi}^{\pi} \mathcal{P}(r, s) d s=0, \forall r \in(0,1)
$$

from (3.1) we obtain

$$
\left\|F_{r}(\cdot)\right\|_{p, \alpha} \leq\left\|F^{+}(\cdot)\right\|_{p, \alpha} \Rightarrow\|F(\cdot)\|_{H_{+}^{p, \alpha}} \leq\left\|F^{+}(\cdot)\right\|_{p, \alpha} .
$$

As a result we have $\|F(\cdot)\|_{H_{+}^{p, \alpha}}=\left\|F^{+}(\cdot)\right\|_{p, \alpha}$.

Let us prove the part $\beta$ ). Let $F(\cdot) \in M H_{+}^{p, \alpha}$. So, for $F(\cdot)$ we have a representation

$$
F_{r}(t)=\frac{1}{2 \pi} \int_{-\pi}^{\pi} F^{+}(s) \mathcal{P}_{r}(r, t-s) d s .
$$

Using the $2 \pi$-periodicity of the function $F^{+}(\cdot)\left(F^{+}(s)=: F^{+}\left(e^{i s}\right)\right)$, we obtain

$$
F_{r}(t)=\frac{1}{2 \pi} \int_{-\pi}^{\pi} \mathcal{P}_{r}(s) F^{+}(t-s) d s .
$$

Consequently

$$
\begin{aligned}
\left\|F_{r}(t)-F^{+}(t)\right\|_{p, \alpha} & =\left\|\frac{1}{2 \pi} \int_{-\pi}^{\pi} \mathcal{P}_{r}(s) F^{+}(t-s) d s-\frac{1}{2 \pi} \int_{-\pi}^{\pi} \mathcal{P}_{r}(s) F^{+}(t) d s\right\|_{p, \alpha} \leq \\
& \leq \frac{1}{2 \pi} \int_{-\pi}^{\pi} \mathcal{P}_{r}(s)\left\|F^{+}(\cdot-s)-F^{+}(\cdot)\right\|_{p, \alpha} d s .
\end{aligned}
$$

Let $\delta>0$-be an arbitrary number. Then from the previous inequality we obtain

$$
\begin{gathered}
\left\|F_{r}(t)-F^{+}(t)\right\|_{p, \alpha} \leq \sup _{|s| \leq \delta}\left\|F^{+}(\cdot-s)-F^{+}(\cdot)\right\|_{p, \alpha} \frac{1}{2 \pi} \int_{|s| \leq \delta} \mathcal{P}_{r}(s) d s+ \\
+\frac{1}{2 \pi} \int_{|s|>\delta} \mathcal{P}_{r}(s)\left\|F^{+}(\cdot-s)-F^{+}(\cdot)\right\|_{p, \alpha} d s .
\end{gathered}
$$

Paying attention to Theorem 2.1 i) and the relation

$$
\left\|F^{+}(\cdot-s)-F^{+}(\cdot)\right\|_{p, \alpha} \leq\left\|F^{+}(\cdot-s)\right\|_{p, \alpha}+\left\|F^{+}(\cdot)\right\|_{p, \alpha}=2\left\|F^{+}(\cdot)\right\|_{p, \alpha},
$$

we have

$$
\left\|F_{r}(t)-F^{+}(t)\right\|_{p, \alpha} \leq \sup _{|s| \leq \delta}\left\|F^{+}(\cdot-s)-F^{+}(\cdot)\right\|_{p, \alpha}+\frac{\left\|F^{+}(\cdot)\right\|_{p, \alpha}}{\pi} \int_{|s|>\delta} \mathcal{P}_{r}(s) d s .
$$

Let $\varepsilon>0$ - be an arbitrary number. Then from $F^{+}(\cdot) \in M^{p, \alpha}$ follows that $\exists \delta_{\varepsilon}>0: \forall \delta \in$ $\left(0, \delta_{\varepsilon}\right)$ the following inequality

$$
\sup _{|s| \leq \delta}\left\|F^{+}(\cdot-s)-F^{+}(\cdot)\right\|_{p, \alpha}<\frac{\varepsilon}{2}
$$


is fulfilled. Take $\forall \delta \in\left(0, \delta_{\varepsilon}\right)$ and fix. Then from Theorem 2.1 iii) follows that $\exists r_{0} \in(0,1)$ : $\forall r \in\left(r_{0}, 1\right)$ :

$$
\frac{\left\|F^{+}(\cdot)\right\|_{p, \alpha}}{\pi} \int_{|s|>\delta} \mathcal{P}_{r}(s) d s<\frac{\varepsilon}{2},
$$

holds. As a result we have

$$
\left\|F_{r}(\cdot)-F^{+}(\cdot)\right\|_{p, \alpha}<\varepsilon, \forall r \in\left(r_{0}, 1\right) .
$$

From the arbitrariness of $\varepsilon>0$ it follows

$$
\lim _{r \rightarrow 1-0}\left\|F_{r}(\cdot)-F^{+}(\cdot)\right\|_{p, \alpha}=0 .
$$

On the contrary, let the relation (3.2) be true. Show that $F^{+}(\cdot) \in M^{p, \alpha}$. We have

$$
\begin{aligned}
& \left\|F^{+}(\cdot-s)-F^{+}(\cdot)\right\|_{p, \alpha} \leq\left\|F^{+}(\cdot-s)-F_{r}(\cdot-s)\right\|_{p, \alpha}+ \\
& \quad+\left\|F_{r}(\cdot-s)-F_{r}(\cdot)\right\|_{p, \alpha}+\left\|F_{r}(\cdot)-F^{+}(\cdot)\right\|_{p, \alpha} .
\end{aligned}
$$

Let $\varepsilon>0$ be an arbitrary number. Then, taking into account the invariance of the norm $\|\cdot\|_{p, \alpha}$ with respect to the shift and relation (3.2), hence we obtain that $\exists r_{0} \in(0,1): \forall r \in$ $\left(r_{0}, 1\right)$ :

$$
\left\|F_{r}(\cdot)-F^{+}(\cdot)\right\|_{p, \alpha}<\frac{\varepsilon}{4} .
$$

For fixed $r \in\left(r_{0}, 1\right)$, the function $F_{r}(\cdot)$ is continuous and $F_{r}(\cdot) \in M^{p, \alpha}$. Then $\exists \delta_{0}>0$ : $\forall \delta \in\left(0, \delta_{0}\right)$ we have

$$
\left\|F_{r}(\cdot-s)-F_{r}(\cdot)\right\|_{p, \alpha}<\frac{\varepsilon}{2}, \forall s:|s| \leq \delta .
$$

As a result we have

$$
\sup _{|s| \leq \delta}\left\|F^{+}(\cdot-s)-F^{+}(\cdot)\right\|_{p, \alpha} \leq \varepsilon,
$$

and it means $F^{+}(\cdot) \in M^{p, \alpha}$. The case ${ }_{m} M H_{-}^{p, \alpha}$ class is proved quite similarly to the case $M H_{+}^{p, \alpha}$. Theorem is proved.

Acknowledgements The author would like to express his deep gratitude to corresponding member of the National Academy of Sciences of Azerbaijan, Professor Bilal T. Bilalov for his attention to this work.

\section{References}

1. Adams, D.R.: Morrey spaces, Springer, (2016).

2. Bilalov, B.T.: The basis property of a perturbed system of exponentials in Morrey-type spaces, Sib. Math. J. 60(2), 249-271 (2019).

3. Bilalov, B.T., Gasymov, T.B., Guliyeva, A.A.: On solvability of Riemann boundary value problem in Morrey-Hardy classes, Turkish J. Math. 40(5), 1085-1101 (2016).

4. Bilalov, B.T., Guliyeva, A.A.: On basicity of exponential systems in Morrey-type spaces, Internat. J. Math. I, 25(6), 1450054 (10 pages)(2014).

5. Bilalov, B.T., Huseynli A.A., El-Shabrawy S.R.: Basis Properties of Trigonometric Systems in Weighted Morrey Spaces, Azerb. J. Math. 9(2), 200-226 (2019). 
6. Daniluk, I.I.: Nonregular boundary value problems on the plane, Nauka, Moscow (1975).

7. Gasymov, T.B., Quliyeva, A.A.: On Basicity of the System of Exponents with Linear Phase in Morrey-Lebesgue Space, Adv. in Anal. 3(2), 113-120 (2018).

8. Guliyeva, F.A., Sadigova, S.R.: On Some Properties of Convolution in Morrey Type Spaces, Azerb. J. Math., 8(1), 140-150 (2018).

9. Guliyev, V.S.: Boundedness of the maximal, potential and singular operators in the generalized Morrey spaces, J. Inequal. Appl. Art. ID 503948, 1-20 (2009).

10. Huseynli, A.A. Mirzoyev, V.S., Quliyeva, A.A.: On Basicity of the Perturbed System of Exponents in Morrey-Lebesgue Space, Azerb. J. Math., 7(2), 197-216 ( 2017).

11. Ismayilova, A.F.: Fractional maximal operator and its commutators on generalized weighted Morrey spaces, Trans. Natl. Acad. Sci. Azerb. Ser. Phys.-Tech. Math. Sci. 39(4), Mathematics, 84-95 (2019).

12. Israfilov, D.M., Tozman, N.P.: Approximation by polynomials in Morrey-Smirnov classes, East journal on approximations, 14(3), 255-269 (2008).

13. Israfilov, D.M., Tozman, N.P.: Approximation in Morrey-Smirnov classes, Azerb. J. Math., 1(1), 99-113 (2011).

14. Kokilashvili, V., Meskhi, A.: Boundedness of maximal and singular operators in Morrey spaces with variable exponent, Armen. J. Math. 1, 18-28 (2008).

15. Kokilashvili V., Meskhi, A., Rafeiro, H., Samko, S.: Integral Operators in NonStandard Function Spaces, Volume 1: Variable Exponent Lebesgue and Amalgam Spaces, Springer (2016).

16. Kokilashvili V., Meskhi, A., Rafeiro, H., Samko, S.: Integral Operators in NonStandard Function Spaces, Volume 2: Variable Exponent Hölder, Morrey-Campanato and Grand Spaces, Springer (2016).

17. Mustafayev R.Ch.: On boundedness of sublinear operators in weighted Morrey spaces, Azerb. J. Math., 2(1), 66-79 (2012).

18. Privalov, I.: Boundary Properties of Analytic Functions [in Russian], Gostekhizdat, Moscow, (1950)

19. Samko, N.: Weighted Hardy and singular operators in Morrey spaces, J. Math. Anal. Appl. 35, 183-188 (2009). 